\title{
Severidad de caries y pérdida de dientes de una población pre-Hispánica del norte de Chile
}

\author{
Caries severity and tooth loss in an ancient pre-Columbian \\ culture in the north of Chile
}

\author{
Urzúa Araya I', Cabello lbacache R ${ }^{1}$, Rodríguez Martínez $\mathrm{G}^{1}$, Gallardo Cortes $\mathrm{S}^{2}$, Sánchez González J3 , Hubbe M
}

\begin{abstract}
RESUMEN
El conocer la historia natural de las patologías bucales sin intervenciones modernas, permite comparar el desarrollo de éstas con los datos actuales. Este estudio tiene como objetivo conocer la severidad de caries dental y de pérdida de dientes en una muestra de restos humanos de la cultura Atacameña (500 DC) en San Pedro de Atacama en el norte de Chile. Se estudiaron 139 restos humanos, en cada uno de ellos se realizó un examen clínico, consignado la información en una ficha individual donde se registró, el número de dientes presentes y perdidos pre y post mortem y el número de lesiones de caries. Estas variables permitieron calcular el índice CPD (Obturadas=0). El análisis estadístico incluyó una descripción de frecuencias y el cálculo de estadísticas de dispersión y tendencia central para las variables continuas. Los resultados muestran que el índice CPD mínimo, asume que las piezas perdidas post mortem estaban sanas fue 17.14 con un promedio de 3.93 lesiones de caries y 13.2 dientes perdidos en vida. Los restos humanos de la población estudiada presentan un gran daño de su salud bucal. Gran cantidad de piezas dentarias perdidas en vida y bajo número de dientes sanos. Rev. Clin. Periodoncia Implantol. Rehabil. Oral Vol. 5(2); 66-68, 2012.
\end{abstract}

Palabras clave: Epidemiología, caries dental, cultura atacameña.

\section{ABSTRACT}

The aim of this study was to determine the severity of dental caries and tooth loss in a sample of 139 human remains of the Atacama culture (500 AD) in San Pedro de Atacama, northern Chile. A clinical examination was performed on each remain by a calibrated examiner. The number of present and missing teeth in pre-and post-mortem stages and the number of carious lesions were recorded. The DMT index (filled $=0$ ) was calculated. Statistical analysis included a description of frequency and calculation of statistical dispersion and central tendency for continuous variables. The results reveal a DMT of 17.14 with an average of 3.93 carious lesions and 13.2 teeth lost in life. The oral health situation of the studied population presented severe damage, high rate of tooth loss and DMT scores.

Rev. Clin. Periodoncia Implantol. Rehabil. Oral Vol. 5(2); 66-68, 2012.

Key words: Epidemiology, dental caries, Atacamenean culture.

\section{INTRODUCCIÓN}

Los estudios epidemiológicos son fundamentales para entender el impacto de una determinada enfermedad, tanto en relación a su importancia clínica como desde el punto de vista de la salud pública. Estos estudios contribuyen a la comprensión de la historia natural de la enfermedad, en este caso, del proceso de caries, y a la determinación de los métodos efectivos para su control y tratamiento ${ }^{(1)}$.

La caries dental es el mayor problema de salud oral en los países industrializados, afectando a la mayoría de los individuos adultos. Además, es la enfermedad bucodental más prevalente en Latinoamérica y en Asia, siendo menos común y severa en países africanos, aún cuando se espera que aumente como resultado de un mayor consumo de azúcares refinados y de una inadecuada exposición a fluoruros ${ }^{(2)}$.

Existe acuerdo general en la existencia de una marcada reducción de prevalencia de lesiones de caries, en la mayoría de los países desarrollados en las recientes décadas ${ }^{(3)}$. Las razones por las cuales se observa esta disminución de la prevalencia de caries es un tema de constante debate, atribuyéndolo a medidas de salud pública como la fluoración del agua potable ${ }^{(1,4)}$, el uso masivo de pastas fluoruradas, el cambio en el criterio diagnóstico y de tratamiento, tendiente hacia la aplicación de medidas preventivas individuales y colectivas, junto con la ejecución de tratamientos con criterios mínimamente invasivos. Adicionalmente, otro factor involucrado en la disminución de la prevalencia de caries, ha sido la mejoría de los estándares socioeconómicos de la población, así como el énfasis que han puesto por los gobiernos en mejorar la salud oral de la población ${ }^{(5,6,7)}$.

Numerosos estudios se han realizado sobre la salud bucal en restos humanos de las culturas pre-Hispánicas ${ }^{(8-11)}$. El desierto de Atacama en el norte de Chile es uno de los lugares que ofrece la oportunidad para estudiar culturas pre-Hispánicas. Este desierto es considerado el más seco del mundo(12). Junto con el clima, el suelo de este lugar presenta condiciones de salinidad y arena que previenen la degradación y juegan un rol importante en la preservación de este patrimonio. En este duro ambiente hay una larga historia de establecimientos humanos, uno de ellos es el oasis de San Pedro de Atacama situado a una altitud de $2.430 \mathrm{mts}$ sobre el nivel del mar, en la confluencia de los ríos San Pedro y Vilama en la región de Antofagasta. Este oasis ha sido habitado durante siglos por la cultura Atacameña los cuales tenían un desarrollo económico en base a pastoreo de camélidos y especialmente al desarrollo agrícola cultivando maíz y algunas plantas locales como el chañar (Geoffroea decorticans) y el algarrobo (Prosopis spp. $)^{(10)}$.

Es importante conocer el desarrollo de la caries dental, con los datos actuales y con evaluaciones históricas, lo que nos permite evaluar la expresión de la enfermedad con estilos de vida pasados, y así conocer la historia natural de la caries dental sin las intervenciones preventivas actuales $^{(8)}$.

El presente estudio tiene como objetivo conocer la severidad

1. Magíster en Ciencias Odontológicas. Área de Cariología, Departamento Odontología Restauradora. Facultad de Odontología, Universidad de Chile. Chile.

2. Cirujano Dentista. Práctica Privada. Chile.

3. Cirujano Dentista. Área de Cariología, Departamento Odontología Restauradora. Facultad de Odontología, Universidad de Chile. Chile.

4. Antropólogo. Instituto de Investigaciones Arqueológicas y Museo. Universidad Católica del Norte. Chile.

Correspondencia autor: Iván Urzúa Araya. ivanurzua@yahoo.com. Sergio Livingstone P. 342 Independencia. Departamento Odontología Restauradora. Facultad de Odontología, Universidad de Chile. Chile. Trabajo recibido el 12/10/2011. Aprobado para su publicación el 03/05/2012. 
de caries dental y de pérdida de dientes en restos humanos de una población adulta de la cultura Atacameña que habitó hace 1.500 años aproximadamente en San Pedro de Atacama en el norte de Chile.

\section{MATERIALES Y MÉTODOS}

Este estudio fue realizado en el Laboratorio de Antropología del Museo Gustavo Le Paige en San Pedro de Atacama durante el año 2009. Fueron seleccionados un total de 139 restos humanos adultos que correspondían a cráneos de individuos que pertenecían a la cultura Atacameña (500 DC) que cumplían con presentar ambos maxilares completos. Estos restos humanos provienen de los cementerios Quitor-6, Coyo-3 y Solcor-3 y se encuentran individualizados y catalogados en el Museo Gustavo Le Paige.

La determinación de la edad de muerte de cada individuo se realizó mediante el análisis de la sínfisis púbica, el grado de sinostosis de las suturas craneanas, patrones de osificación, análisis de los huesos largos y el grado de erupción de las piezas dentales de acuerdo a la metodología descrita en la literatura ${ }^{(13)}$.

La determinación del sexo fue realizada por marcadores en la pelvis de los restos humanos de acuerdo a los criterios descritos por Bass (1987) y Buikstra y Ubelaker (1994) $)^{(13,14)}$.

La determinación del sexo y la edad de muerte fueron realizados por antropólogos expertos del Museo Gustavo Le Paige.

El examen bucal de cada uno de los restos humanos se realizó por un examinador entrenado previamente, utilizando luz ambiental del laboratorio que aseguraba una adecuada visibilidad. Para el examen se utilizó un espejo plano número 5 y una sonda de caries con extremo romo. Para cada uno de los restos humanos, se realizó una ficha individual y un odontograma donde se registró, el número de dientes presentes, el número y ubicación de lesiones de caries de acuerdo a los criterios de la Organización Mundial de la Salud (OMS) ${ }^{(15)}$ y los dientes perdidos en vida y post mortem, dependiendo si el alvéolo encontraba cicatrizado o no ${ }^{(8)}$.

Esta información permitió calcular el índice CPD mínimo, que corresponde al número de dientes afectados por caries o perdidos por la misma razón, asumiendo que las piezas dentarias perdidas post mortem estaban sanas y el CPD máximo asumiendo que las piezas dentales perdidas post mortem estaban con lesiones de caries. STATA 10.

Los datos fueron tabulados y analizados con el software estadístico

El análisis incluyó una descripción de frecuencias y el cálculo de estadísticas de dispersión y tendencia central para las variables continuas.

\section{RESULTADOS}

Los resultados obtenidos del análisis de los restos humanos estudiados muestran un promedio de edad de 28.4 años (DE 8.39). Este promedio ha sido calculado sobre la base del $23 \%$ de los individuos, dado que el $77 \%$ de la muestra esta identificado como individuos con dentición permanente pero sin determinación de la edad. La estimación del género está presentada en la Tabla 1, donde se aprecia que en la mayoría de los individuos no fue posible la determinación del sexo.

Tabla 1. Distribución por sexo de la muestra de restos humanos.

\begin{tabular}{|c|c|c|}
\hline Género & $\mathbf{n}$ & $\begin{array}{c}\text { Proporción } \\
\text { (\%) }\end{array}$ \\
\hline Mujer & 19 & 13.7 \\
\hline Hombre & 19 & 13.7 \\
\hline No Definido & 101 & 72.7 \\
\hline Total & 139 & 100 \\
\hline
\end{tabular}

La severidad de caries y sus componentes se observan en la Tabla 2, el CPD mínimo, el cual asume que las piezas perdidas postmortem estaban sanas y el CPD máximo que asume que las piezas dentales perdidas postmortem estaban afectadas por caries.
Tabla 2. Severidad de caries y sus componentes.

\begin{tabular}{|c|c|c|c|c|c|c|}
\hline $\mathbf{n}$ & $\begin{array}{c}\text { CPD } \\
\text { Mínimo }\end{array}$ & $\mathbf{C}$ & $\mathbf{P}$ & $\begin{array}{c}\text { CPD } \\
\text { Máximo }\end{array}$ & $\mathbf{C}$ & $\mathbf{P}$ \\
\hline 139 & $\begin{array}{c}17.14 \\
(\mathrm{DE} 9.4)\end{array}$ & $\begin{array}{c}3.93 \\
(\mathrm{DE} \mathrm{3.15)})\end{array}$ & $\begin{array}{c}13.2 \\
(\mathrm{DE} \mathrm{10.06)})\end{array}$ & $\begin{array}{c}20.85 \\
(\mathrm{DE} 9.2)\end{array}$ & $\begin{array}{c}7.65 \\
(\mathrm{DE} \mathrm{4.73)}\end{array}$ & $\begin{array}{c}13.2 \\
(\mathrm{DE} 10.06)\end{array}$ \\
\hline
\end{tabular}

En el Gráfico 1 se muestra la distribución por frecuencia de los dientes afectados por caries y perdidos tanto pre mortem como post mortem. Se puede observar que los molares corresponden al tipo de diente que se encuentra mas afectado y con las mayores frecuencias de dientes perdidos. Los datos sugieren una distribución con imagen especular entre el lado derecho e izquierdo, a nivel de molares y con un leve predominio de mayor daño en las piezas inferiores.

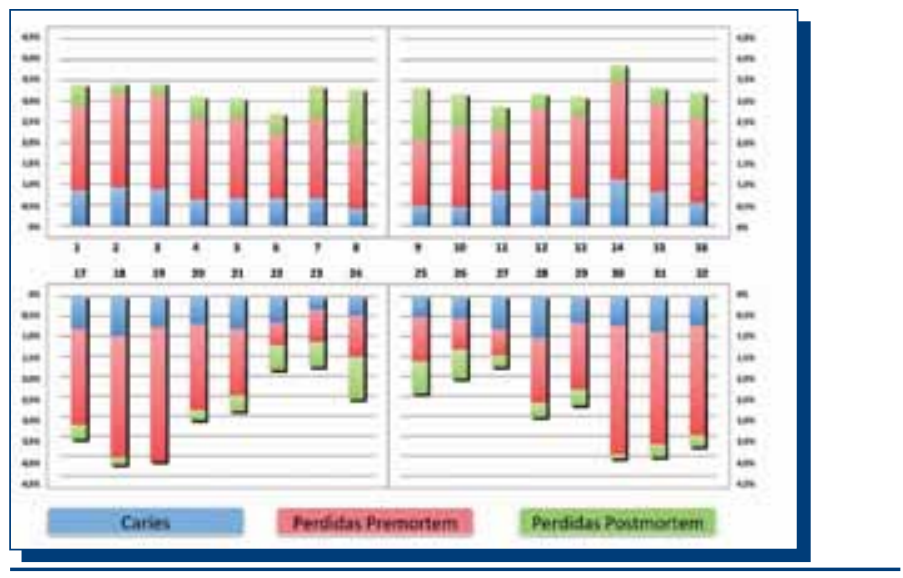

Gráfico 1.

\section{DISCUSIÓN}

Las buenas condiciones de los restos humanos permitieron una adecuada detección de las lesiones de caries. Se pudo observar un importante daño por caries en la dentición de la muestra estudiada. Es importante destacar que se debe tener cautela en el análisis de los datos dado que se desconocen las circunstancias exactas en que los individuos enferman o las reales causas de la perdida de dientes. Sin embargo, este escenario representa una oportunidad única de estudiar la caries dental.

La relación entre la dieta y caries esta bien documentada, encontrándose que una alta ingesta de hidratos de carbono en general, es responsable de una alta prevalencia de caries, como lo observado en este estudio. Trabajos realizados por Cucina y Tiesler (2003), en la cultura Maya ${ }^{(9)}$, muestran una alta prevalencia de caries en esta población lo que es atribuido a la ingesta de una dieta en base a maíz, situación similar a lo reportado por Meller y cols. (2009), para la cultura Pica-Tarapacá(8).

La cultura Atacameña, reportada en este articulo, tenía una alimentación basada principalmente en el consumo de maíz, este hecho podría explicar la alta prevalencia de caries y pérdida de dientes encontrada en esta población.

En los países industrializados se ha observado una declinación en la prevalencia de lesiones de caries en los últimos años, lo que se ha atribuido al cuidado en la salud bucal de la población junto al uso de fluoruros, a pesar de que no se ha reportado un cambio en los patrones de alimentación ${ }^{(16)}$.

Comparando esta tendencia con lo encontrado en este estudio se puede observar que un estilo de vida en base a una alimentación refinada rica en hidratos de carbono y en ausencia de medidas de control del proceso de caries se traduce en una alta prevalencia de esta patología.

Por otro lado, estudios realizados en poblaciones medievales, antes del ingreso de azúcar a Europa, muestran una baja prevalencia de caries en esas poblaciones a pesar de no contar con medidas de control de caries $^{(17,18)}$.

Para el cálculo del CPD mínimo (17.14) se asumió que todos los dientes perdidos post mortem estaban sanos, lo que puede dar una visión más optimista de las condiciones bucales del grupo en estudio y para el cálculo del CPD máximo (20.85) se asumió que todos los dientes 
perdidos post mortem estaban con lesiones de caries, lo que da la visión más pesimista de las condiciones bucales del grupo en estudio.

Considerando que el promedio de lesiones de caries presentes es relativamente bajo (3.93) creemos que el CPD mínimo es mas cercano a la realidad que el CPD máximo, ya que este último podría sobre estimar el numero de lesiones de caries.

Si comparamos estos datos con los encontrados por Meller y cols. $(2009)^{(8)}$ en una población Pre Hispánica de la cultura Pica-Tarapacá del norte de Chile (1000 DC), observamos que el promedio de lesiones de caries fue de 2.1, el promedio de dientes perdidos por cada individuo en vida era de 9 dientes y el índice CPD (Obturadas $=0$ ) mínimo fue de 12.9. Esto podría sugerir que vemos una disminución en la expresión de la enfermedad, si se compara 500 DC con 1000 DC. Sin embargo, este análisis debe ser cuidadoso dado que existen variables asociadas a los estilos de vida de los individuos que no han sido consideradas.

Por otro lado, si observamos estos resultados en conjunto con estudios de poblaciones actuales como el de Gamonal (1996) ${ }^{(19)}$ realizado en adultos de 35-44 años de edad en Santiago, vemos que el índice COPD reportado para ese estudio fue de 25.96 el cual experimentó un aumento de 5 unidades a lo reportado en este estudio en el índice CPD máximo. El aumento del COPD en el contexto actual está influenciado por el componente obturadas y por el aumento del número de lesiones de caries de 9.4 reportado por Gamonal en el año 1996 a 3.93 registrado en este estudio. No cabe duda que la comparación de los resultados de este estudio con estudios actuales no es posible dada las diferencias mayores en la metodología empleada. Sin perjuicio de lo anterior, la descripción de estos antecedentes contribuye a entender la expresión de la enfermedad en el pasado y en la actualidad.

La distribución porcentual de lesiones de caries y pérdida de dientes en vida del grupo de restos humanos en estudio, nos muestra que las piezas mas afectadas son los primeros y segundos molares lo cual concuerda con los estudios realizados en poblaciones medievales en Francia y Corea ${ }^{(20,21)}$ y con estudios longitudinales considerados clásicos en cariología como los de Carlos y Gittelsohn (1965)(22).

\section{CONCLUSIONES}

Los restos humanos de la población estudiada presentan un importante daño de su salud bucal, una gran cantidad de piezas dentarias perdidas en vida y bajo número de dientes sanos.

\section{CONFLICTOS DE INTERÉS}

Los autores declaran no tener conflictos de interés.

\section{REFERENCIAS BIBLIOGRÁFICAS}

1. Fejerskov O, Kidd E. Dental caries: The disease and its clinical management. 2008 Second edition, Wiley-Blackwell.

2. Petersen PE. The World Oral Health Report 2003. Continuous improvement of oral health in the $21^{\text {st }}$ century-the approach of the WHO Global Oral Health Program. http://www.who.int/oral_health/media/en/ orh_report03_en.pdf. Accesado 12-5-2011.

3. Petersson HG, Bratthall D. The caries decline: A review of reviews. Eur J Oral Sci, 1996; 104: 436-443.

4. Seppä L, Kärkkäinen S, Hausen H. Caries trends 1992-1998 in two low-fluoride finnish towns formerly with and without fluoridation. Caries Res, 2000; 34: 462-468.

5. Nadanowsky P, Sheiham A. Relative contribution of dental services to the changes in caries levels of 12-year-old children in 18 industrialized countries in the $1970^{\text {s }}$ and early $1980^{\text {s. }}$. Commun Dent Oral Epidemiol, 1995; 23: 331-339.

6. Konig K. Clinical manifestations and treatment of caries from 1953 to global changes in the 20 centuty. Caries Res, 2004; 38(3): 168-172.

7. Marthaler T. Changes in dental caries 1953-2003. Caries Res, 2004; 38(3): 173-181.

8. Meller C, Urzúa I, Moncada G, von Ohle C. Prevalence of oral pathologic findings in an ancient pre-Columbian archeologic site in the Atacama desert. Oral Diseases, 2009; 15: 287-294.

9. Cucina A, Tiesler V. Dental caries and ante-mortem tooth loss in the Northern Peten area, Mexico: A biocultutal perspective on social status differences among the Classic Maya. Am J Phys Anthropol, 2003; 122: $1-10$.

10. Costa MA, Neves WA, Hubbe M. Influencia de Tiwanaku en la calidad de vida biológica de la población prehistórica de San Pedro de Atacama. Estudios Atacameños, 2004; 103-116.

11. Rodríguez CD, Rodríguez EL. Analysis of dental samples from buried individuals in "El Morro de Tulcan", pre-Hispanic pyramid from Popayan, Colombia. Enlaces, 2000; 3(3): 26-32.
12. Clarke JDA. Antiquity of aridity in the Chilean Atacama Desert. Geomorphology, 2006; 73: 101-111.

13. Bass W. Human osteology: A laboratory and field manual of the human skeleton. Editor M.K.Trimble, Missouri Arch Society, Columbia. 1987.

14. Buikstra JE, Ubelaker DH. Standards for data collection from human skeletal remains. Fayetteville: Arkansas Archaeological Survey, Series 44. 15. WHO 1997. Oral healh surveys: Basic methods, $4^{\text {th }}$ ed. World Health Organization: Geneva.

16. Bratthall D, Hansel-Petersson G, Sundberg H. Reasons for the caries decline: What do the experts believe? Eur J Oral Sci, 1996; 104: 416-422. 17. Varrela TM. Prevalence and distribution of dental caries in a late medieval population in Finland. Arch Oral Biol, 1991; 36: 553-559.

18. Watt ME, Lunt DA, Gilmour WH. Caries prevalence in the permanent dentition of a mediaeval population from the south-west of Scotland. Arch Oral Biol, 1997; 42: 601-620.

19. Gamonal J. Prevalencia de enfermedades periodontales y de caries dental en la población de 35-44 y 65-74 años de nivel socioeconómico bajo y medio bajo de la provincia de Santiago, Región Metropolitana y determinación de los recursos necesarios para su tratamiento. Tesis para optar al grado de Magíster en Ciencias Odontológicas. Facultad de Odontología Universidad de Chile, 1996.

20. Esclassan R, Astie F, Sevin A, Donat R, Lucas S, Grimoud AM. Study of the prevalence and distribution of dental caries in a medieval population in Southwest France. Rev Stomatol Chrir Maxillofac, 2008 Feb; 109(1): 28-35.

21. Han SS, Baek KW, Shin MH, Kim J, Oh C, Lee SJ, Shin DH. Dental caries prevalence of medieval Korean people. Arch Oral Biol, $2010 \mathrm{Jul}$ 55(7): 535-540.

22. Carlos JP, Gittelsohn AM. Longitudinal studies of the natural history of caries: A life-table study of caries incidence in the permanent teeth. Arch Oral Biol, 1965 Sep-Oct; 10(5): 739-751. 